\title{
CUADERMQQM
}

\section{DESARROLLO LOCAL A PARTIR DEL USO DE TECNOLOGÍA SOCIAL: UN ENFOQUE ALTERNATIVO}

\begin{abstract}
Paula Peyloubet
Dra. Arquitecta. Investigadora adjunta del CONICET (Consejo Nacional de Investigaciones Científicas y Técnicas) Investigadora del Centro Experimental de la Vivienda Económica. Docente en la cátedra de Introducción a la Tecnología de la Facultad de Arquitectura-Universidad Nacional de Córdoba. Directora del Instituto de Tecnología y Vivienda de la Facultad de Arquitectura-Universidad Católica de Córdoba.
\end{abstract}

\section{Héctor Massuh}

Arquitecto. Investigador Principal del CONICET. Director del CEVE (Centro Experimental de la Vivienda Económica).

\section{Tomás O’Neill}

Arquitecto. Contratado de AVE-CEVE. Integrante del área de Investigación en el Centro Experimental de la Vivienda Económica. Docente en la cátedra de Introducción a la Tecnología de la Facultad de Arquitectura-Universidad Nacional de Córdoba.

\section{Valeria Fenoglio}

Arquitecta. Becaria de posgrado de CONICET. Integrante del área de investigación en el Centro Experimental de la Vivienda Económica. Desarrollo de Tesis Doctoral en la Facultad de Arquitectura-Universidad Nacional de Tucumán.

\section{Gabriela Valladares}

Licenciada en Biología. Becaria de posgrado de CONICET. Integrante del área de Investigación en el Centro Experimental de la Vivienda Económica. Desarrollo de Tesis Doctoral en la Facultad de Ciencias Químicas-Universidad Nacional de Córdoba.

\section{Institución CEVE}

Centro Experimental de la Vivienda Económica. Unidad Ejecutora de CONICET. Con sede en la Ciudad de Córdoba, cuenta con más de 40 años de experiencia en desarrollo de tecnologías constructivas y de gestión abordando la problemática de hábitat desde una visión integral. www.ceve.org.ar 


\title{
Artículos Arbitrados
}

CUADERNO URBANO. Espacio, Cultura, Sociedad - VOL. 9 - № 9 (Octubre 2010) pp. 169-191. ISNN 1666-6186

\begin{abstract}
Resumen
La problemática social en la Argentina requiere de un cambio de visión en la construcción de la resolución del problema. El fenómeno de la exclusión y el empobrecimiento de grandes sectores de la población pone en crisis los enfoques que se vienen utilizando para el abordaje de dicha problemática y muestra una ineficiencia en las soluciones planteadas, como también la ausencia de acciones transformadoras de la realidad social. Los modelos de desarrollo, imperantes en el sistema económico mundial, se basan en un juego de oferta y demanda en el que el libre mercado se autorregula dando lugar a crecimientos inequitativos y decrecimientos fabulosos. Se parte, entonces, por reconocer la ausencia de modelos no excluyentes alternativos. El presente trabajo pretende exponer una experiencia de investigación-acción cuyo enfoque de resolución de la problemática se plantea a partir de procesos inclusivos aplicando el uso de tecnologías sociales como instrumento para el desarrollo local. Caso: Villa Paranacito.
\end{abstract}

\section{Palabras clave}

Desarrollo local, Tecnología Social, Innovación Social, Circuito Productivo Interactoral.

\section{Abstract}

The social problems of Argentina require a change of vision in the construction of the solution to these problems. The phenomena of exclusion and impoverishment of large sectors of the population demonstrate the need to change the approaches that are being used to face these problems as they are inefficient in terms of the solutions they generate which do not help to produce transforming actions regarding social reality. The prevailing, world-wide, economic system development models, are based on the game of supply and demand where the free market supposedly regulates itself. As we know, this leads to inequitable growth and fabulous socio-economic declines; the absence of non-excluding alternative models.is recognized. This paper presents a case of empirical action research whose approach to solving the problem arises from applying inclusive processes using social technologies as a tool for local development. Case: Villa Paranacito.

\section{Keywords}

Local Development, Social Technology, Social Innovation, Productive Interactoral Circuit. 
Peyloubet, P. Massuh, H. O’Neill, T. Fenoglio, V. Valladares, G.

DESARROLLO LOCAL A PARTIR DEL USO DE TECNOLOGÍA SOCIAL: UN ENFOQUE ALTERNATIVO

\section{INTRODUCCIÓN}

Las expresiones aquí vertidas son el corolario de diversas investigaciones y acciones realizadas en los últimos diez años por un equipo interdisciplinario de investigación del CEVE¹, en la localidad de Villa Paranacito, Entre Ríos. Su campo de acción se encuentra referido a desarrollos tecnológicos de producción sustentable de casa partes de madera para la producción social del hábitat.

Los proyectos de investigación realizados consideran diversas líneas prioritarias, como el desarrollo y la innovación tecnológica, líneas que surgen como respuesta al reconocimiento de un problema instalado en la zona del Delta de Paraná y que se encuentra relacionado con un ineficiente uso de un recurso natural renovable, como es la madera de álamo. Paralelamente a esta problemática se presenta otra situación deficitaria relacionada con la producción de hábitat para sectores de escasos recursos que no acceden a vivienda propia o sufren hacinamiento.

En este contexto, los proyectos de investigación aquí presentados tienen por objetivo el fortalecimiento del perfil productivo del lugar, la necesidad de vivienda y de trabajo a partir de la implementación de un Circuito Productivo Interactoral de casa partes de madera. Éste se basa en el uso del recurso natural (madera de álamo), de los recursos socio culturales y del aprovechamiento de la infraestructura de producción existente, procurando la inclusión de sectores de escasos recursos en la economía formal de la localidad, con lógicas de producción posibles para esta inclusión. Es decir que la investigación propone la exploración de nuevos procesos de producción mixta en los que intervienen diversos actores: Municipio, Carpintería municipal, Escuela Técnica, Mypes (pequeñas y medianas empresas), Familias necesitadas, Organismos de Ciencia y Tecnología.

Dicho Circuito Productivo Interactoral de casa partes de madera se inscribe en el marco del desarrollo local, ya que su implementación y construcción responden a ciertas condiciones que son necesarias para el desarrollo de sociedades: es endógeno: ya que se implementa de abajo hacia arriba y el municipio cumple un rol estratégico; descubre yefectiviza todo el potencial productivo que tienen las localidades: recurso natural y humano; es participativo: porque trabajan todos los niveles sociales en la resolución del problema; se centra en el conocimiento: especialmente el conocimiento práctico resolviendo la problemática a partir de la propia gente y procura construir unaeconomía más solidaria eigualitaria (CoRAGGIO, 2004). 


\section{Artículos Arbitrados}

CUADERNO URBANO. Espacio, Cultura, Sociedad - VOL. 9 - № 9 (Octubre 2010) pp. 169-191. ISNN1666-6186

2- Tomamos el concepto de tecnología Social (TS) desarrollado particularmente en el marco de la Red de Tecnología Social de Brasil. El surgimiento de la TS surge en esta última década en Brasil, donde actores diversos preocupados por la creciente exclusión social precarización e informalización del trabajo in formalización del trabajo y el aumento de la pobreza, aun con crecimiento del PBI del país, concertan la necesidad de construir una alternativa eficaz para la solución de estos problemas sociales.
A lo largo de los años la investigación se ha ido adhiriendo a nuevos conceptos. En este sentido, queremos expresar mediante este trabajo la adhesión al concepto de Tecnología Social ${ }^{2}$. Concepto nuevo y en construcción, que nace como alternativa a la tecnología convencional. El uso de tecnologías sociales como instrumento para el desarrollo se convierte en el enfoque alternativo que queremos exponer en el presente trabajo. En este sentido, el Circuito Productivo Interactoral utiliza para su construcción el componente indispensable de las tecnologías sociales que es la participación directa de la comunidad en la resolución de los problemas, basándose en la existencia de potencialidades en ella y que representan efectivas soluciones de transformación social.

\section{PRESENTACIÓN DEL PROBLEMA}

La problemática social en la Argentina requiere de un cambio de visión en la construcción de la resolución del problema. El fenómeno de la exclusión y el empobrecimiento de grandes sectores de la población pone en crisis los enfoques y estrategias que se vienen utilizando para el abordaje de dicha problemática y muestra una ineficiencia en las soluciones planteadas, como también la ausencia de acciones transformadoras de la realidad social.

Las tendencias mundiales caracterizadas por el proceso de globalización de los mercados, la política de ajuste estructural y las nuevas políticas sociales han configurado - y siguen haciéndolo - en nuestro país como en el resto de América Latina un contexto de profunda crisis socio-económica de una buena parte de nuestra población. En este sentido, el modelo neoliberal de economía capitalista, imperante en nuestro país, refleja la inaptitud del mercado para promover desarrollo, ya que da lugar a crecimientos inequitativos y decrecimientos fabulosos y genera en el campo popular la precarización de sus condiciones de vida.

Como problema en la transformación de la situación planteada, de extrema pobreza y exclusión, se advierte por un lado la ausencia de modelos inclusivos que generen cambios profundos, y por otro, la presencia de estrategias y enfoques que atienden las consecuencias del problema y no las causas. La principal causa del aumento de la pobreza en nuestro país es la gran desigualdad en la distribución de la riqueza e ingresos. Frente a este modelo excluyente, el abordaje del problema no puede ser otro que trabajar por el desarrollo de la sociedad desde una perspectiva integral; con una fuerte mirada socio-económica, cultural y política. 
Peyloubet, P. Massuh, H. O’Neill, T. Fenoglio, V. Valladares, G. DESARROLLO LOCAL A PARTIR DEL USO DE TECNOLOGÍA SOCIAL: UN ENFOQUE
ALTERNATIVO

Adoptar una mirada integral implica reconocer la articulación real entre economía, sociedad, cultura y política, y es el nivel local la situación privilegiada para comprender y actuar frente al problema, atendiendo a las reales necesidades de una demanda explícita que se expresa con claridad en los contextos locales.

En este sentido, los procesos socio-espaciales relacionados con el acceso a la ciudad y las formas de producción de hábitat —especialmente la de los sectores más pobresderecho a la ciudad y la vivienda son esencialmente cuestiones locales. Es fundamental trabajar por modelos de desarrollo no excluyentes desde los espacios locales. En este contexto se reconoce el problema desde nuestro campo de acción, que es el hábitat. La política propuesta por el Estado, en materia de hábitat, es actualmente centralizada y no considera las características locales de cada región, tanto en su dimensión urbana y social como productiva laboral de recursos naturales y saberes populares. Esta situación de predominio nacional genera en los gobiernos locales una insuficiente capacidad administrativa y organizacional, ya que no pueden controlar efectivamente las acciones para resolver los reales problemas locales. Por lo tanto, estas decisiones supra-territoriales subordinan los procesos sociales, culturales y urbanos del territorio local, como así también las posibles instancias de participación. Es justamente este asunto el que queremos poner en controversia, ya que la problemática del hábitat requiere de un abordaje complejo, a partir de la explícita referencia de lo local y la participación interactoral en la toma de decisiones.

Cuando se hace referencia a la producción local de hábitat, el término incluye no sólo a la dimensión físico espacial, sino, de manera interdisciplinaria e integral, a las dimensiones socio culturales, productivo laborales, políticas, económicas y ambientales de la localidad en cuestión, que es lo que la hace singular y única a dicha producción. Es así que, para abordar la problemática aquí planteada desde nuestro campo de acción que es el hábitat, se asume como postura su comprensión de manera integral. La resolución de la problemática abordada no se construye desde un producto (por ejemplo: vivienda), sino a través de la implementación de un Circuito Productivo Interactoral que a partir del uso de tecnologías sociales contribuye al desarrollo local.

\section{MARCO TEÓRICO - CONCEPTUAL}

Para el abordaje del tema problema, la presente investigación asume la existencia de marcos conceptuales a los que adhiere. Dichos conceptos preconcebidos ayudarán a comprender 


\section{Artículos Arbitrados}

CUADERNO URBANO. Espacio, Cultura, Sociedad - VOL. 9 - № 9 (Octubre 2010) pp. 169-191. ISNN1666-6186

3- Lo local no hace referencia al desarrollo autónomo de cada localidad, sino que expresa la importancia de generar desarrollo desde abajo, donde se explicitan las reales necesidades de la gente, para luego generar sinergia alcanzando escala a partir gia las articulaciones de las localidades en redes socio-productivas. el enfoque propuesto y argumentarán el proceso de construcción de nuevo conocimiento a partir de la implementación de un Circuito Productivo Interactoral como modelo alternativo para contribuir al desarrollo local ${ }^{3}$.

Se presenta en primer lugar el concepto de Desarrollo Local. El desarrollo local no puede ser otra cosa que el desarrollo de una economía, una sociedad y un sistema político locales, condiciones básicas para una mejora sostenida en la calidad de vida de sus ciudadanos (Coraggio, 2004). Es decir, la búsqueda de desarrollo económico, social, político y cultural que fomente todo el potencial productivo que tienen las ciudades y que está en la sociedad y sus organizaciones. El desarrollo tiene como factor clave de su estructuración a la propia población local.

El autor también expresa la necesidad de trabajar en el desarrollo de la sociedad centrado en dos dimensiones: la economía y el conocimiento. La economía entendida como la organización de la producción, del trabajo y la innovación, así como también las múltiples formas de resolución de las necesidades de la gente (CoRAGGIO, 2004). Con esta concepción de la economía como dimensión necesaria para el desarrollo, el conocimiento también aparece como recurso en el que los distintos actores sociales intervienen de manera activa en la resolución de los problemas sociales. Es decir, permite centrar el conocimiento científico y técnico con el conocimiento práctico que tienen las sociedades.

En segundo lugar, con respecto al desarrollo de la Tecnología, hasta hoy ha sido manejada como una caja negra, como una esfera autónoma y neutral que determina su propio camino de desarrollo, generando inexorables efectos constructivos o destructivos a su paso (THOMAs, 2009). A esta visión determinista de la tecnología se contraponen nuevas vías de construcción y resolución de problemas socio-técnicos, y son las llamadas tecnologías sociales, que nacen como alternativa a la Tecnología Convencional. Dicha Tecnología Convencional, hoy imperante, genera en el modelo vigente juegos de mercadotecnia de los que se benefician unos pocos y se excluye a muchos.

En la década del 60 y 70 surge el concepto de Tecnología Apropiada, que intenta sumar la población excluida a la lógica de mercado y de renta de la TC. Los esfuerzos y buena voluntad por reclamar un espacio en este escenario permitieron poner en marcha, con cortos alcances, una visión integral de necesidades y demandas de los sectores pobres de la 
Peyloubet, P. Massuh, H. O’Neill, T. Fenoglio, V. Valladares, G.

DESARROLLO LOCAL A PARTIR DEL USO DE TECNOLOGÍA SOCIAL: UN ENFOQUE
ALTERNATIVO
ALTERNATIVO

sociedad. Sin embargo, el gran equívoco imperante en ese momento fue que el juego de oferta y demanda se instaló con un nuevo nombre, consagrando dentro de la TA la transferencia tecnológica (TT). La TT, de la mano de la TA, trajo consigo la idea de que las necesidades de los sectores pobres y excluidos podían ser resueltas por el sector de producción cognitiva y ofrecidas "generosamente" para su uso. En ese camino se desestimó el propio sector beneficiario como potencial colaborador de la resolución. El fracaso dio lugar a la idea de que los problemas no podían ser postulados a priori ni a partir del reconocimiento del problema por otros sectores no afectados. Así fue sustituida la ingenua e ineficaz idea de "oferta" y "demanda" o "transferencia tecnológica" producida por la comunidad de investigadores por una construcción colectiva de conocimiento no excluyente, que incorpora los valores, intereses, necesidades y saberes de los sectores pobres (Peyloubet, 2008).

En este sentido y en el marco del desarrollo local centrado en el conocimiento, nos adherimos a las nuevas ideas que se gestan y que relacionan la tecnología con el desarrollo social. Con una lógica de tecnología como construcción social consideramos que para abordar el problema de la pobreza, la tecnología social se constituye en una estrategia clave para nuestras sociedades. Para eso es necesaria la inclusión de las comunidades que generen soluciones tecno-productivas que sean adecuadas al territorio donde se construyen. Se entiende por Tecnología Social - aun siendo un concepto en construcción- a "los productos, técnicas y/o metodologías reaplicables desarrolladas en la interacción con la comunidad y que representan efectivas soluciones de transformación social" (DAGNINO, 2004). Por lo tanto, las tecnologías sociales se constituyen en tecnologías no excluyentes, ya que permiten el acceso igualitario a bienes y servicios para toda la población y también la participación de la comunidad en general en los procesos de diseño y toma de decisiones para su implementación.

Otro concepto relacionado con la presente investigación es el de Innovación. La contribución de la teoría de la innovación como marco teórico, a pesar de que su real aplicación no fue positiva, fue fundamental para la superación de los defectos del modelo cognitivo que sirvió de sustrato para el concepto de TA. Esta teoría criticó el poco realismo del modelo de "oferta y demanda" para comprender el producto "conocimiento", y propuso para eso una perspectiva basada en la interacción de actores desde un primer momento, para engendrar, en función de múltiples criterios, un conocimiento (bienes o servicios) que pudiera ser utilizado en el propio lugar e incorporado en su sistema de saberes 


\section{Artículos Arbitrados}

CUADERNO URBANO. Espacio, Cultura, Sociedad - VOL. 9 - № 9 (Octubre 2010) pp. 169-191. ISNN 1666-6186

(Peyloubet, 2008). Entonces, la teoría de la innovación permitió pensar a la Tecnología Social como un proceso de innovación con características particulares. Desde la perspectiva de lo antes dicho se puede decir que la Innovación Social - mediante el enfoque de la Tecnología Social - es un concepto utilizado para hacer referencia al "conocimiento intangible incorporado a las personas que tienen por objetivo el aumento de la efectividad de los procesos, servicios y productos relacionados a la satisfacción de las necesidades sociales, referidos a valores, estilos de desarrollo, proyecto nacional social, político, económico y ambiental" (DAGNINO, 2004).

\section{MARCO IDEOLÓGICO}

Para comenzar a construir una alternativa es necesario reconocer la negatividad del modelo de desarrollo hoy vigente. Las teorías neoclásicas, que tienen un claro predominio en el diseño de las políticas económicas, se constituyen como el modelo dominante. Dichas teorías cultivan mecanismos de mercado que sólo generan mayores concentraciones del poder y brutales desigualdades socio-económicas.

En este sentido, en nuestras sociedades aparece el conflicto y aparece la lucha. Por un lado están las fuerzas que intentan imponer un sentido común que legitime ese modelo imperante, y por otro existen las fuerzas que plantean la necesidad de enfoques alternativos; modelos que generen espacios donde los constructos sociales sean cooperativos y solidarios, espacios de participación igualitaria, de acciones distributivas basadas en la inclusión social.

Esta última fuerza es el escenario de lucha cultural que asume esta investigación, ya que para generar cambios profundos y modificaciones en este sistema de dominio hay que trabajar sobre la cultura, sobre el sentido común. Para Gramsci el sentido común es una concepción del mundo mecánicamente impuesta por una criatura extraña, y agrega que el poder de influencia que tiene la ideología dominante es visible en el contenido del sentido común (Alfaro, 2009). Si el sentido común de nuestra sociedad está impregnado por los valores del mercado - un mercado que excluye y no participa a todos por igual-, hay que trabajar para cambiar ese sentido, y es la lucha por el sentido de la participación. El problema de la participación no es la falta de ella, sino su calidad en los sectores populares (CORAGGIO, 2004). 
Peyloubet, P. Massuh, H. O’Neill, T. Fenoglio, V. Valladares, G. DESARROLLO LOCAL A PARTIR DEL USO DE TECNOLOGÍA SOCIAL: UN ENFOQUE
ALTERNATIVO

En este sentido, la presente investigación asume que la mejora en la calidad de vida de las poblaciones en general debe ser una construcción colectiva en la que se empodere a los que no tienen poder, se escuche a quienes hablan también en la lengua del silencio y se respeten las condiciones culturales diversas con la convicción profunda de creer que éstos son los argumentos básicos para poner en marcha la mencionada calidad de vida (Peyloubet, 2008).

A partir de la experiencia de Villa Paranacito, se intenta construir un nuevo conocimiento mediante modelos contra-hegemónicos que inicien procesos no excluyentes con la participación y el conocimiento de todos los actores sociales, y así poder consolidar el derecho a una mejor calidad de vida de toda la población.

Por lo tanto, abordar el problema a partir de la construcción de modelos de desarrollo alternativos centrados en la economía y el conocimiento a través de tecnologías sociales es lo que pretende expresar el presente trabajo. Una economía como sistema de resolución de necesidades, un conocimiento horizontalizado y una tecnología al servicio de todos constituyen el nuevo paradigma que queremos construir, el escenario de lucha cultural presente en nuestras investigaciones.

\section{EXPERIENCIA EN VILLA PARANACITO: CIRCUITO PRODUCTIVO INTERACTORAL}

Como materialización de lo expresado hasta el momento, se presentará el caso de Villa Paranacito (Entre Ríos), que es producto de cuatro proyectos consecutivos de investigación-acción que se vienen trabajando a partir del año 1998, cuando sucedieron las inundaciones en el litoral argentino. En el caso de Villa Paranacito la inundación alcanzó aproximadamente $2,00 \mathrm{~m}$ de cota máxima, con una ocupación del agua en la ciudad y aledaños por espacio de más de nueve meses ininterrumpidos.

El trabajo del equipo comenzó entonces, cuando aquella inundación tomó el territorio, llevando a cabo una solución de urgencia que consistió en la unión de dos líneas de trabajo que nuestra institución viene trabajando hace 40 años como metodología para abordar la problemática de hábitat desde una visión integral. Por un lado se puso en marcha un proyecto de investigación que requirió de una construcción de conocimientos tecnológicos 


\section{Artículos Arbitrados}

CUADERNO URBANO. Espacio, Cultura, Sociedad - VOL. 9 - № 9 (Octubre 2010) pp. 169-191. ISNN1666-6186

4- PID (Proyecto de Investigación y Desarrollo) 23121: diseño e implementación de un Circuito Productivo Interactoral de casa partes de madera que contribuya al fortalecimiento del perfil productivo del lugar, en marco del desarrollo local

5- PICT (Proyecto de Investigación de Ciencia y Tecnología) 670. Estudio teórico empírico de especies maderables no convencionales para su aplicación en la construcción de vivienda. Caso: Álamo. y de un enfoque epistemológico alternativo para dicha construcción, y por otro se puso en marcha una acción que materializó los desarrollos tecnológicos para realizar el primer programa de viviendas palafíticas con base en recursos locales, tanto humanos como naturales, que promoviera la articulación de actores para el fortalecimiento de un desarrollo local.

A partir de esta experiencia el equipo de investigación quedó vinculado con la localidad y sus actores, el municipio, la escuela técnica, los productores forestales y la población en general. La confianza adquirida a partir de la tarea desarrollada, el reconocimiento de un recurso natural renovable y la problemática en la producción social del hábitat fueron los factores de formulación de otros proyectos, como fue el proyecto PID $23121^{4}$ finalizado recientemente y el proyecto PIC $670^{5}$ que se encuentra en curso. Este último se relaciona con el reconocimiento profundo de la madera de álamo para otorgar nuevos usos de la producción forestal que generen valor agregado a esta producción y promuevan el uso sustentable de los recursos naturales con capacidad de generar empleo en dicha región.

Los objetivos de los proyectos están relacionados con el fortalecimiento de la producción integral del hábitat social, y procuran la capacitación laboral y la generación de trabajo a partir de los recursos socio-culturales y del aprovechamiento de la infraestructura existente en la localidad. En ese marco y con esos objetivos, los proyectos procuran la exploración de nuevos procesos de producción mixta en los que intervienen diversos actores que configuran un Circuito Productivo Interactoral de gestión y producción local que responda al fortalecimiento del desarrollo desde lo local. Los proyectos de investigación proponen el desarrollo de un nuevo proceso organizacional de producción a partir de productos tecnológicos: casa partes, que conforman componentes para vivienda de madera, pudiendo constituir un sistema constructivo para vivienda en sí mismo o componentes adaptables para tecnología de vivienda tradicional, dando respuestas a la demanda de vivienda nueva como de mejoramiento.

Por lo tanto, la presente investigación asume el siguiente presupuesto: es posible que la construcción de un Circuito Productivo Interactoral, como modelo alternativo, a partir del uso de tecnologías sociales, opere como herramienta necesaria para contribuir al desarrollo local.

A continuación se describirá el momento empírico de la investigación a partir de la formulación e implementación del Circuito Productivo Interactoral en la localidad. Su descripción se realizará en función de las condiciones que son necesarias para el desarrollo local. 
Peyloubet, P. Massuh, H. O’Neill, T. Fenoglio, V. Valladares, G.

DESARROLLO LOCAL A PARTIR DEL USO DE TECNOLOGÍA SOCIAL: UN ENFOQUE
ALTERNATIVO
ALTERNATIVO

\section{Desarrollo Endógeno}

Una de la condiciones del desarrollo local es que debe ser endógeno, es decir, de abajo hacia arriba. En este sentido, los gobiernos municipales juegan un rol muy importante para el desarrollo local. En nuestro país hemos experimentado un proceso de descentralización que consistió, básicamente, en que el Estado nacional transfirió a las provincias y éstas a los municipios una serie de responsabilidades. Si bien ciertas acciones y decisiones todavía siguen siendo tomadas por el Estado nacional, mostrando una subordinación en los procesos sociales, culturales y urbanos del territorio local, consideramos la necesidad de trabajar para fortalecer aquellas responsabilidades transferidas a los municipios, ya que es en los contextos locales donde se producen de manera explícita las reales necesidades, especialmente de los más pobres. Entonces, si el desarrollo local debe ser endógeno - es decir, desde adentro o abajo-, el nivel local es la situación privilegiada para comprender y actuar frente a la problemática en la producción social del hábitat.

La municipalidad de Villa Paranacito es un organismo cuya actividad política tiene 20 años de antigüedad. Gran parte de la población laboralmente activa está empleada en el Estado municipal. El municipio cuenta con un equipo de técnicos y carpinteros que forman parte de la carpintería municipal. Desde el período de las inundaciones y de la intervención del equipo de investigación, la entidad muestra voluntad para recibir propuestas que puedan aportar a resolver la problemática socio-habitacional, como así también la situación socio-productiva. El municipio reconoce la necesidad de vivienda nueva o mejoramiento, como así también la necesidad de valorar y diversificar el uso del recurso forestal para la construcción de viviendas en madera de álamo y así contribuir al desarrollo socio-económico, político y cultural de la localidad.

El municipio se constituyó en un actor clave dentro del circuito productivo. Su posicionamiento como adoptante-socio permitió llevar adelante la producción de casa partes, en madera de álamo, a través de su financiamiento. Desde el inicio, participó en la formulación del proyecto, los procesos de gestión como en el desarrollo tecnológico (producto: casa partes de madera) y construcción de las viviendas.

\section{Potencial Productivo}

El desarrollo económico y social implica descubrir y efectivizar todo el potencial productivo que tienen las localidades, es decir, un desarrollo ligado con los sistemas de producción. 


\section{Artículos Arbitrados}

CUADERNO URBANO. Espacio, Cultura, Sociedad - VOL. 9 - № 9 (Octubre 2010) pp. 169-191. ISNN 1666-6186

La región productiva donde se localiza Villa Paranacito corresponde al delta del Paraná. Dicha región se configura como el marco físico más importante en el cultivo de álamos y sauces de nuestro país (salicáceas). Dichas especies proveen un amplio rango de productos de la madera (madera rolliza, postes, pulpa y papel, tableros, madera terciada, madera contrachapada, madera aserrada, embalaje, muebles, etc.), productos no maderables (forraje, leña) y servicios (abrigo, sombra y protección de suelos, agua, cultivos, ganado y viviendas).

Se reconoce como problema, en la zona del delta del Paraná, el ineficiente uso de un recurso natural renovable: madera de salicáceas. En la actualidad, la madera de salicáceas producida en la región se destina principalmente para triturado y en mucha menor proporción para aserrado y debobinado. Aproximadamente el 90 \% de esta madera tiene como objetivo el triturado, y por lo tanto no se le realizan los manejos necesarios para darle mayor calidad en campo, cerrando posibilidades de mercado. Esta situación impone la necesidad de planificar y diversificar la producción forestal del sector y su uso procurando aumentar su sustentabilidad como actividad económica productiva. Dicha mono-producción tampoco genera trabajo suficiente en la región, ya que sólo emplea mano de obra para la producción primaria, dejando un vacío importante en el empleo secundario en el que se agregaría valor a la materia prima.

Reconocido el problema, uno de los objetivos que plantean los proyectos de investigación, en el orden tecnológico, es colaborar en la redefinición del uso que actualmente se hace del recurso maderable (embalajes, cajonería fruti-hortícola, apícola, funeraria, etc.) impulsando una diversificación hacia la producción industrializada de "casa partes", a partir de la validación de la madera como elenco de materiales para la construcción, como aporte alternativo a la solución del déficit habitacional y al fortalecimiento de la economía local del sector, trabajo forestal industrial con valor agregado en origen, reconvirtiendo la forma de producción, implementando procesos con criterio industrial operario intensivo; es decir, no capital intensivo, sino generador de puestos de trabajo con alta productividad, rescatando de la cultura habitacional del isleño (habitante del delta) el empleo del álamo en la construcción artesanal de las viviendas.

Por otro lado, el re-direccionamiento de la materia prima hacia una producción de ciclo tecnológico distributivo como es la construcción propiciaría circuitos productivos con mayor 
Peyloubet, P. Massuh, H. O’Neill, T. Fenoglio, V. Valladares, G.

DESARROLLO LOCAL A PARTIR DEL USO DE TECNOLOGÍA SOCIAL: UN ENFOQUE
ALTERNATIVO
ALTERNATIVO

inserción social y generación de empleo diversificado, e impactaría entonces sobre las actividades productivas de la región, la dinámica económica y el desarrollo social.

A lo largo del proyecto se han realizado visitas a productores de plantaciones de álamo en Villa Paranacito. Una de las problemáticas que genera preocupación en ellos es la plaga llamada Taladro de los Forestales (Megaplatypus mutatus). Dicha plaga es en la actualidad la más importante de la especie álamo en Argentina y provoca una merma en los volúmenes útiles de madera por hectárea, debido a la quebradura de los fustes por efecto del viento a la altura de las galerías, y en el volumen de madera de calidad en los árboles en pie, especialmente cuando el destino de la madera es el aserrado o el debobinado (CASAUBON, 2006).

En este sentido y en el marco del proyecto en curso PICT 670 (estudio teórico empírico de especies maderables), se desarrolla un doctorado con el siguiente tema: diseño de matrices poliméricas biodegradables para la liberación controlada de feromonas en el control de insectos plaga: Megaplatypus mutatus (taladro de los forestales). Dicho doctorado propone como control de plagas un método a partir de la utilización de feromonas sexuales para el control de insectos problema. Mediante el uso de feromonas sexuales se puede atraer al macho o a la hembra de la especie, y una vez que tal individuo se aproxima al árbol es capturado por medio de trampas elaboradas para tal fin. De esta manera, al no poder reproducirse los individuos capturados, el número de potenciales transportadores de la plaga disminuye. Con el uso de esta técnica de control de plagas es posible eliminar el uso de insecticidas con las siguientes ventajas: son biodegradables por ser productos naturales y no contaminan el ambiente; se usan en muy pequeñas cantidades; son específicas para cada especie, por lo que controlan a una población determinada, evitando matar a otras especies que podrían ser benéficas. De esta manera se obtienen dos beneficios: por un lado se logra controlar la plaga problema, causante de pérdidas económicas importantes para los productores forestales y por otro lado se logra un control amigable con el ambiente sin afectar a especies que conviven en el mismo lugar y que no ocasionan pérdidas ni producen problemas en las forestaciones.

\section{Participación}

Otra condición para el desarrollo es la participación. El problema de la participación no es su falta, sino su calidad en los sectores populares (Coraggio, 2004:35). Por lo tanto, 


\section{Artículos Arbitrados}

CUADERNO URBANO. Espacio, Cultura, Sociedad - VOL. 9 - № 9 (Octubre 2010) pp. 169-191. ISNN 1666-6186

6- SECYT: Secretaría de Ciencia y Tecnología. CONICET: Consejo Nacional de Investigación Científica y Técnicas. CEVE: Centro Experimental de la Vivienda Económica. para poner en marcha la mejora en la calidad de vida de las poblaciones y en especial de los sectores más populares, es necesario adoptar como estrategia espacios de construcción colectiva donde se involucre a todos los sectores sociales a participar de manera activa en la resolución de los problemas locales. En este sentido, la articulación Interactoral es el componente clave del circuito productivo para el desarrollo local. El proyecto PID 23121, mediante la construcción del circuito, involucró la participación de diversos actores locales, y generó interrelaciones sectoriales de singular importancia, como fueron el sector político: municipio; el sector educativo: la escuela técnica; el sector productivo: los productores de madera, los aserraderos, las mypes, el sector de la población: familias con necesidad de vivienda y el sector de cienciay tecnología: SECYT-CONICET-CEVE ${ }^{6}$.

Dicho Circuito Productivo Interactoral de casas partes de madera construyó espacios de vinculación y concertación de los diferentes actores sociales para la producción social del hábitat y la generación de empleo a nivel local. La participación de los distintos actores se realizó a lo largo de todo el proceso de construcción del circuito, tanto en el desarrollo tecnológico de casa partes de madera como en la toma de decisiones políticas, técnicas, etc.

Actores que participaron del circuito Ciencia y Tecnología: el actor CEVE se constituyó como la institución beneficiaria y su adoptante fue el Municipio de Villa Paranacito. Las actividades iniciales del proyecto tuvieron su escenario en la sede de CEVE, donde se realizaron los primeros ensayos del prototipo de madera. Luego el escenario se trasladó a Villa Paranacito, a la escuela técnica. Allí se realizaron varios encuentros productivos de manera progresiva, en los que se logró una buena participación y permitieron incorporar al desarrolo inicial variantes aportadas por los alumnos y maestros carpinteros de la escuela.

Desarrollo de la primera mini serie del prototipo. Luego se puso en marcha la producción de la serie, pero a escala de casa partes y el montaje de vivienda nueva. Para dicho proceso el municipio ingresó en el proyecto en la formulación teórica del Circuito Productivo Interactoral y en la producción de dichas casa partes a través de sus técnicos carpinteros. La participación de este actor presentó las mismas características que la escuela, ya que se incorporaron al desarrollo tecnológico los saberes de los técnicos y el producto fue nuevamente formulado para su construcción final.

La escuela técnica: Villa Paranacito posee una escuela de Educación Técnica, en creciente desarrollo, cuyas especialidades son las de Carpintería y Metal-mecánica. La capacidad 
Peyloubet, P. Massuh, H. O’Neill, T. Fenoglio, V. Valladares, G. DESARROLLO LOCAL A PARTIR DEL USO DE TECNOLOGÍA SOCIAL: UN ENFOQUE ALTERNATIVO

técnica, docente y organizativa del cuerpo de docentes que la llevan adelante les ha permitido crecer y obtener subsidios mediante la presentación de proyectos que posibilitaron actualizar maquinarias de taller y equipar la sala de informática. La escuela técnica se reconoce como un actor importante dentro del circuito, encargado de desarrollar conjuntamente y transferir los conocimientos a la población. Dicho motivo fue el que llevó a incorporarla como actor social relevante dentro del circuito. Se realizaron cinco encuentros productivos entre el equipo de investigadores de CEVE y los alumnos y docentes de la escuela técnica. (Figuras 3 y 4 )

Municipio de Villa Paranacito; Técnicos municipales: el municipio intervino en el proyecto a través de recursos humanos y financieros. Participó en la formulación y desarrollo de la producción tecnológica y gestión del proyecto. (Figuras 5 y 6 )

Carpintería municipal: el equipo de técnicos y carpinteros que forman parte de la carpintería municipal llevaron adelante la producción de casa partes en madera de álamo. Se realizaron varios talleres entre el equipo de CEVE y los técnicos municipales. En dicho proceso se aprovechó al máximo el aporte de la experiencia y saberes de los carpinteros municipales al producto tecnológico. (Figuras 7 y 8 )

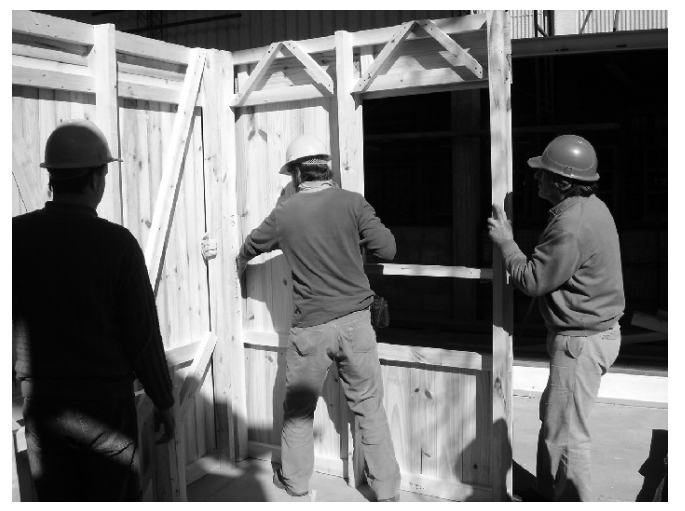

Desarrollo tecnológico-sede CEVE Fuente: proyecto PID 23121

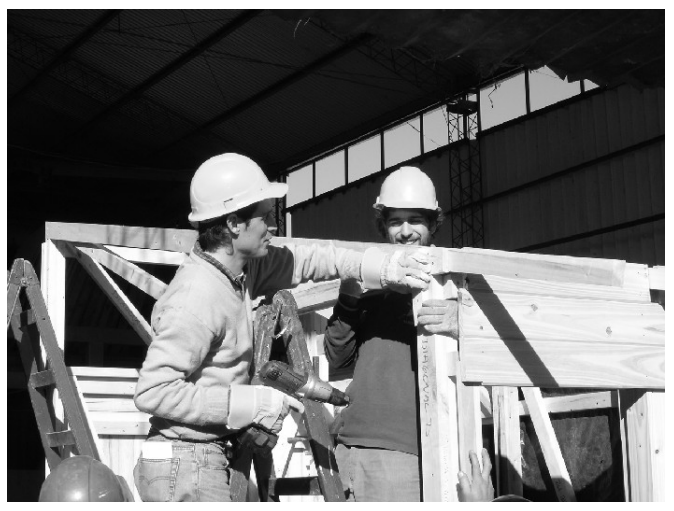

Desarrollo Tecnológico-sede CEVE Fuente: proyecto PID 23121 


\section{Artículos Arbitrados}

CUADERNO URBANO. Espacio, Cultura, Sociedad - VOL. 9 - № 9 (Octubre 2010) pp. 169-191. ISNN 1666-6186
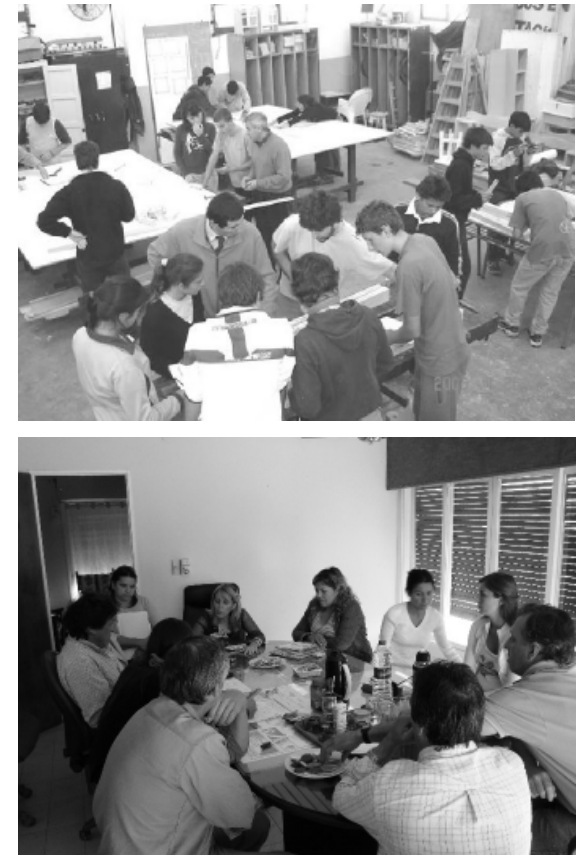

2 -3-Encuentros productivos con escuela Técnica Fuente: proyecto PID 23121

Técnicos albañiles municipales: el municipio de Villa Paranacito fue el encargado de la construcción de la obra húmeda de las cinco viviendas en el Cerro Poblacional. Se construyeron cinco plateas de hormigón armado y cinco núcleos húmedos. En dichos núcleos se utilizaron los bloques de cemento que el mismo municipio produce. (Figuras 9 y 10 )

Bloquera municipal: la municipalidad de Villa Paranacito cuenta con una planta de producción de bloques cementicios. En el proyecto se utilizaron los bloques de cemento para la construcción del sector húmedo de las viviendas con el objetivo de incorporar un producto que la misma localidad produce y que en términos de costos es más accesible. (Figuras 11 y 12)

Herrería municipal: en el desarrollo tecnológico del prototipo de vivienda, los paneles contemplan una serie de sujeciones de chapa (flejes) que unen los encuentros de la estructura en madera. El actor encargado de la realización de estos flejes fue la herrería municipal. (Figuras 13 y 14)

Aserraderos: Paranacito ha sido desde siempre un poblado cuya especialidad ha girado alrededor de la explotación forestal. Existe en la zona una verdadera cultura maderera y un deseo de continuar con esa explotación. Paranacito posee dos

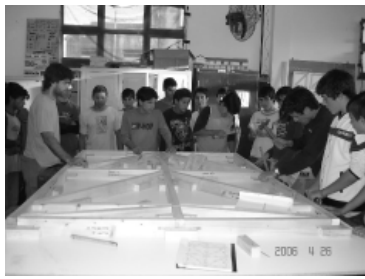

4 -5-Reuniones con técnicos municipales Fuente: proyecto PID 23121

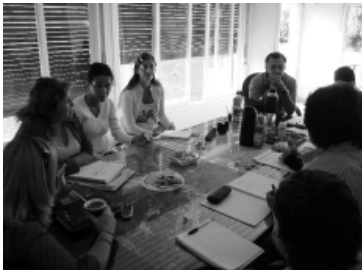

\section{(1)}

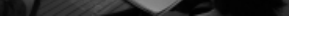

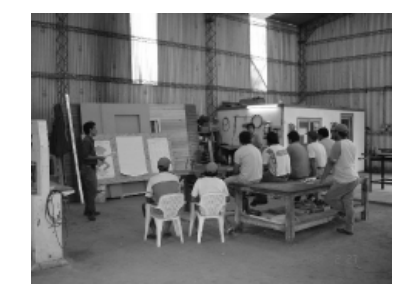

$\begin{array}{lll}7 & \text { y } 8 \text {-Encuentros productivos con carpinteros }\end{array}$

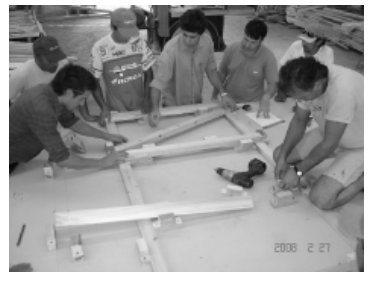

municipales. Fuente: proyecto PID 23121 
Peyloubet, P. Massuh, H. O’Neill, T. Fenoglio, V. Valladares, G. DESARROLLO LOCAL A PARTIR DEL USO DE TECNOLOGÍA SOCIAL: UN ENFOQUE ALTERNATIVO

aserraderos de pequeña envergadura. El primero de ellos está dentro de los límites de la planta urbana, el segundo se encuentra sobre la ruta de acceso a la ciudad y de dimensiones menores. Ambos aserraderos, Piñol y Kirpach, participaron del proyecto mediante la provisión de tablas de madera de álamo, tanto para los ensayos como para la construcción de las cinco viviendas. Durante el desarrollo del proyecto los dos aserraderos han manifestado la importancia del Circuito Productivo Interactoral en la localidad, como así también la voluntad de destinar parte de su producción a la construcción de viviendas. (Figuras 15 y 16)

Productores forestales: durante el proyecto se han realizado visitas a tres productores de plantaciones de álamo en Villa Paranacito. A partir de entrevistas se pudo conocer la situación de sus producciones: datos de la situación actual de producción, los modos de comercialización, la problemática de los incendios y las inundaciones que afectan a la región, como así también los tipos de plagas que afectan a la madera. Cada productor adopta diferentes soluciones frente a dichas problemáticas. En las visitas realizadas los productores mostraron el interés por el Circuito Productivo Interactoral,

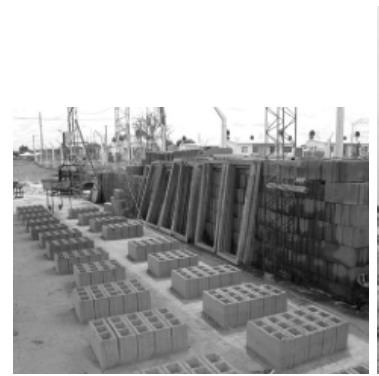

11 y 12 Bloquera municipal.Construcción con bloques de cemento Fuente: proyecto PID 23121
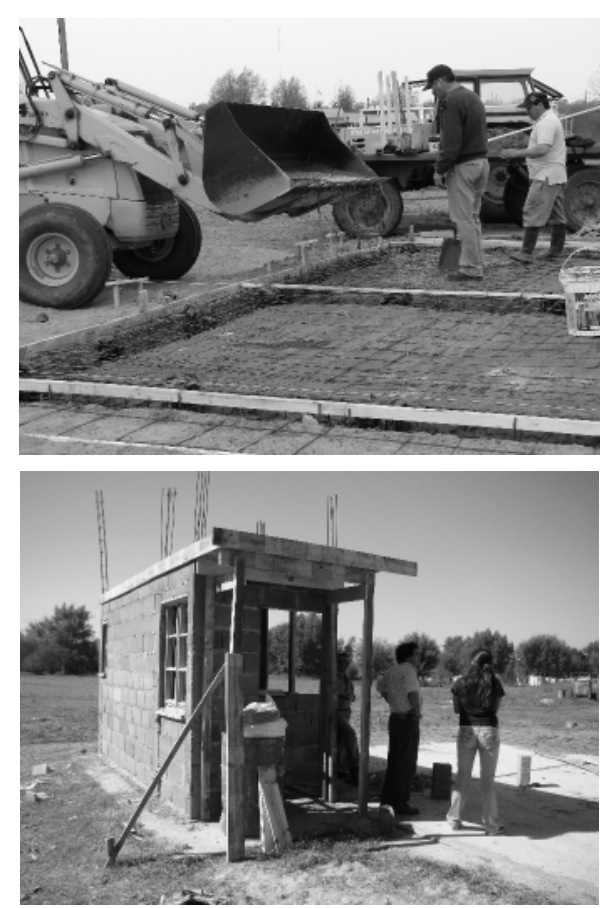

9 y 10-Construcción vía húmeda de la vivienda Fuente: proyecto PID 23121

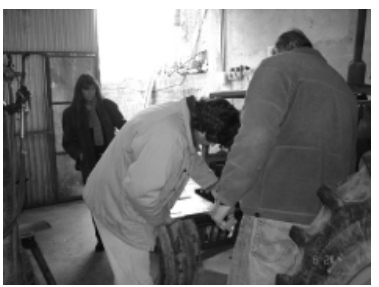

13 y 14- Herrería municipal

Fuente: proyecto PID 23121

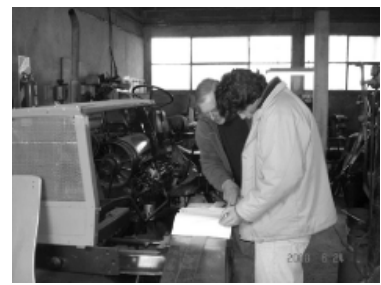

$\sqrt{2}$ 


\section{Artículos Arbitrados}

CUADERNO URBANO. Espacio, Cultura, Sociedad - VOL. 9 - № 9 (Octubre 2010) pp. 169-191. ISNN 1666-6186
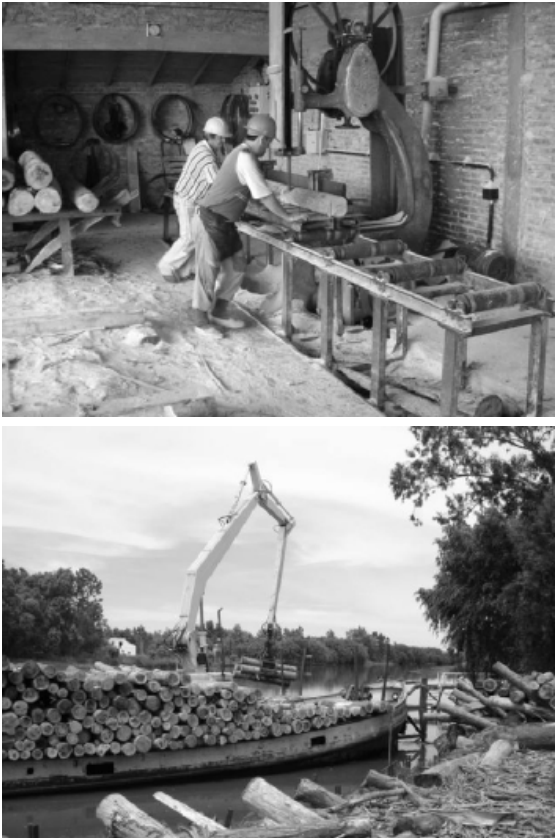

15 y 16- Aserraderos locales Fuente: proyecto PICT 670 como así también la necesidad de diversificar el destino de la madera de álamo, hoy destinada en su mayoría a pasta para papel. (Figuras 17 y 18 )

Familias beneficiadas: la producción tecnológica de la vivienda en madera de álamo se destinó a cinco familias de la localidad. Las familias participaron en el proceso no desde la auto-construcción sino desde la toma de decisiones. Con ese objetivo se desarrollaron diversos talleres participativos en los que cada familia pudo elegir su lote, elegir hacia dónde proyectar el crecimiento de su vivienda, tomar decisiones sobre la disposición del núcleo húmedo, etc. También se realizaron varios encuentros cuyos temas estaban relacionados con el mantenimiento de las viviendas, asesoramiento para la compra de ciertos materiales o talleres de consulta que se realizaban directamente en el sitio donde se estaban construyendo las viviendas. (Figuras 19 y 20)

\section{Conocimiento}

El desarrollo está centrado en el conocimiento, no sólo técnico y científico sino también aquel cargado de significaciones culturales, saberes y prácticas populares instaladas en todas las comunidades. La posición epistemológica que asume este trabajo comprende al problema, desde un nuevo planteamiento cognitivo y cultural basado en la capacidad creativa

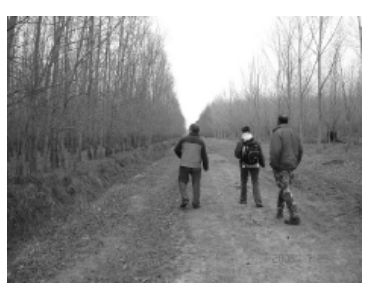

17 y 18 -Visita a la producción forestal Fuente: proyecto PICT 670

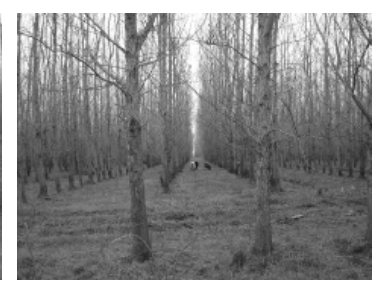

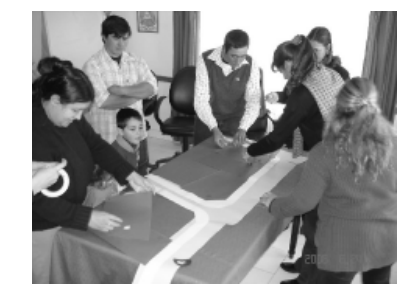

19 y 20- Encuentros Participativos Fuente: proyecto PID 23121

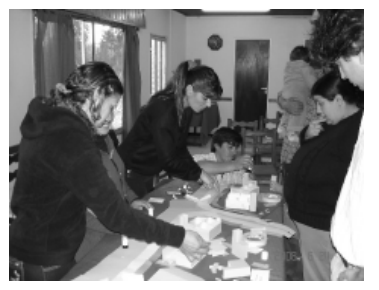

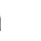


Peyloubet, P. Massuh, H. O’Neill, T. Fenoglio, V. Valladares, G.

DESARROLLO LOCAL A PARTIR DEL USO DE TECNOLOGÍA SOCIAL: UN ENFOQUE
ALTERNATIVO
ALTERNATIVO

del investigador (sujeto cognoscente) y de la participación activa del ciudadano (sujeto por conocer) (VASILACHIS, 2006).

Este nuevo enfoque alternativo potencia los saberes de la gente, y su participación directa en la resolución de los problemas es sin dudas una salida.

Con este enfoque epistémico y en relación con la construcción social del hábitat, el Circuito Productivo Interactoral procura abordar la problemática. A modo de marco referencial, el sector educativo en nuestro país durante la década de los 90 asistió al deterioro de la Enseñanza Técnica de Nivel Medio, y generó un déficit de personal capacitado en oficios. Un país en crecimiento demanda la capacitación de sus habitantes. Es menester impulsar la recuperación de esta enseñanza. La capacitación y el conocimiento posibilitan el desarrollo de los individuos. Entre Ríos es la cuarta provincia Argentina con mayor cantidad de establecimientos de educación técnica del país, por encima de la provincia de Mendoza y después de los centros más poblados del país. En este contexto, la escuela de Villa Paranacito se convirtió para el proyecto en el actor clave para aprovechar el recurso humano y potenciar el intercambio de conocimiento en la localidad. La interacción con los distintos actores permitió el intercambio de saberes y de cultura en el trabajo de la madera, como así también los saberes culturales propios de los pobladores. A partir de la posición investigativa de este enfoque, el conocimiento es horizontalizado y la transferencia tecnológica como modo de operar deja de existir para pasar a ser una tecnología co-construida, componente de las tecnologías sociales.

\section{Economía solidaria e igualitaria}

Otra condición del desarrollo local es la construcción de una economía más igualitaria y más solidaria. El proyecto procura revalorizar y diversificar el uso de un recurso natural a través de la generación de una red de actores (circuito) que de manera igualitaria y solidaria intentan generar nuevas formas de producción y de organización, para resolver así las reales necesidades de la localidad.

\section{METODOLOGÍA}

Queremos expresar mediante el presente trabajo que la metodología planteada en la formulación del proyecto proponía la transferencia tecnológica, es decir, el investigador a 


\section{Artículos Arbitrados}

CUADERNO URBANO. Espacio, Cultura, Sociedad - VOL. 9 - № 9 (Octubre 2010) pp. 169-191. ISNN 1666-6186

\begin{tabular}{|c|c|c|c|c|}
\hline & \multicolumn{2}{|c|}{ CIRCUITO PRODUCTIVO INTERACTORAL: TECNOLOGIAA SOCIAL } \\
\hline & & & ACTORES & PRODUCTO TECNOLÓGICO \\
\hline \multirow{16}{*}{ 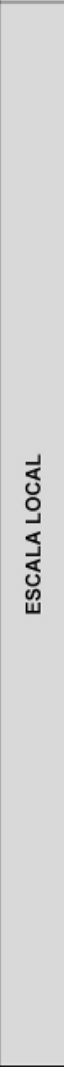 } & \multirow{3}{*}{ 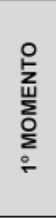 } & \multirow{3}{*}{ 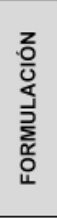 } & $\begin{array}{c}\text { Ave-Ceve-SSDUV-CONICET- } \\
\text { ANPCYT }\end{array}$ & $\begin{array}{l}\text { En base a las tareas realizadas en villa } \\
\text { Paranacito desde el año } 1998 \text { se continua } \\
\text { trabajando con el municipio y la comunidad } \\
\text { en general y se decide iniciar un proyecto } \\
\text { PID }\end{array}$ \\
\hline & & & $\begin{array}{l}\text { Ave-Ceve-Municipio de Villa } \\
\text { Paranacito, Escuela Técnica }\end{array}$ & $\begin{array}{l}\text { Formulación del proyecto de manera } \\
\text { conjunta en la localidad de Villa Paranacito }\end{array}$ \\
\hline & & & $\begin{array}{l}\text { Ave-Ceve-Municipio de Villa } \\
\text { Paranacito }\end{array}$ & $\begin{array}{l}\text { Presentación del proyecto en ANPCyT. } \\
\text { Aceptación y aprobación }\end{array}$ \\
\hline & \multirow{5}{*}{ 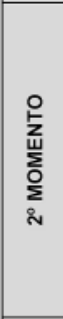 } & \multirow{5}{*}{ 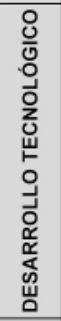 } & \multirow{5}{*}{$\begin{array}{l}\text { Ave-Ceve- Escuela Técnica } \\
\text { de Villa Paranacito/ } \\
\text { Aserradero Local Kirpach }\end{array}$} & $\begin{array}{l}\text { Primer desarrollo tecnológico de } \\
\text { componentes. Producción de mini serie en } \\
\text { la sede de CEVE. }\end{array}$ \\
\hline & & & & $\begin{array}{l}\text { Primer encuentro productivo con la Escuela } \\
\text { Técnica. Primer mini serie en Villa } \\
\text { Paranacito. }\end{array}$ \\
\hline & & & & $\begin{array}{l}\text { Reformulación del desarrollo tecnológico. } \\
\text { Primer prototipo en sede CEVE. }\end{array}$ \\
\hline & & & & $\begin{array}{l}\text { Cuatro Encuentros productivos mas se } \\
\text { realizaron la escuela. }\end{array}$ \\
\hline & & & & $\begin{array}{l}\text { En el quinto encuentro se realizó una } \\
\text { simulación del prototipo en la escuela } \\
\text { técnica en Villa Paranacito. }\end{array}$ \\
\hline & \multirow{5}{*}{ 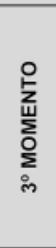 } & \multirow{5}{*}{ 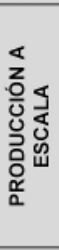 } & \multirow{3}{*}{$\begin{array}{c}\text { Ave-Ceve- Municipio: } \\
\text { Hacienda Carpintería } \\
\text { municipal, Mype (Chavéz), } \\
\text { Aserradero local (Piñol), } \\
\text { Herrería municipal, bloquera } \\
\text { municipal } \\
\end{array}$} & $\begin{array}{l}\text { Varios encuentros productivos con los } \\
\text { técnicos carpinteros. }\end{array}$ \\
\hline & & & & $\begin{array}{l}\text { Reformulación del primer prototipo } \\
\text { diseñado en la escuela con técnicos } \\
\text { municipales }\end{array}$ \\
\hline & & & & Segundo prototipo \\
\hline & & & \multirow{2}{*}{$\begin{array}{l}\text { Productores forestales, } \\
\text { Bióloga de Ceve, IMBIV, } \\
\text { CPEIN, CONICET }\end{array}$} & $\begin{array}{l}\text { Producción forestal. Estudio de plagas de } \\
\text { álamo }\end{array}$ \\
\hline & & & & $\begin{array}{l}\text { Mantenimiento y preservación de la madera } \\
\text { aserrada }\end{array}$ \\
\hline & \multirow{3}{*}{ 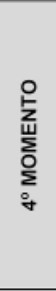 } & \multirow{3}{*}{ 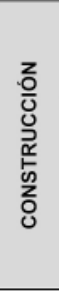 } & $\begin{array}{c}\text { Ave-Ceve, Municipio, } \\
\text { Albañilerla, Carpintería, } \\
\text { Electricidad, Escuela Técnica } \\
\text { (alumnos y docentes) }\end{array}$ & $\begin{array}{l}\text { Construcción de cinco viviendas. } \\
\text { Replanteo, plateas, Núcleo húmedo, } \\
\text { montaje via seca, terminaciones }\end{array}$ \\
\hline & & & Familias beneficiadas & $\begin{array}{l}\text { Talleres participativos. Toma de decisiones } \\
\text { de las familias en aspectos constructivos y } \\
\text { habitacionales de la vivienda como asi } \\
\text { también la elección del terreno. }\end{array}$ \\
\hline & & & Ave-Ceve, SSDUV & $\begin{array}{l}\text { Solicitud del CAT: Certificado de aptitud } \\
\text { técnica como herramienta necesaria para el } \\
\text { municipio en la construcción de planes de } \\
\text { vivienda con madera de álamo. }\end{array}$ \\
\hline 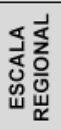 & \multicolumn{2}{|c|}{$\begin{array}{l}\text { MOMENTO } \\
\text { ACTUAL }\end{array}$} & Ave-Ceve & $\begin{array}{l}\text { Escalar el circuito Productivo Interactoral } \\
\text { mediante un proyecto a escala regional. }\end{array}$ \\
\hline
\end{tabular}


Peyloubet, P. Massuh, H. O’Neill, T. Fenoglio, V. Valladares, G.

DESARROLLO LOCAL A PARTIR DEL USO DE TECNOLOGÍA SOCIAL: UN ENFOQUE
ALTERNATIVO
ALTERNATIVO

partir de un desarrollo tecnológico transfiere sus conocimientos a ciertos destinatarios. A lo largo del proyecto y a partir de la interacción con los diferentes actores de la localidad de Villa Paranacito, la metodología fue adquiriendo un carácter participativo en el que el conocimiento no fue transferido sino co-construido; empoderando a todos los actores locales, tanto en la toma de decisiones como en el desarrollo tecnológico. (Cuadro l)

\section{DESDE LO LOCAL A LO REGIONAL: UN CAMBIO DE ESCALA}

A partir de la descripción del caso Villa Paranacito y la experiencia construida allí, el equipo de investigación continúa trabajando en la temática de modelos de desarrollo intersectoriales e interactorales y procura la construcción de insumos para la elaboración de un modelo tecnológico de Gestión y Producción Interactoral ${ }^{7}$ basado en el fortalecimiento del perfil productivo local, la generación de empleo y el fortalecimiento de la producción social del hábitat. La articulación interactoral e intersectorial se presenta como indispensable en la elaboración del modelo. Esto significa que los distintos actores sociales deben intervenir activamente en la construcción conceptual y de conocimiento para atender las demandas sociales. Dicho modelo se propone como replicable y flexible en otras localidades.

La adhesión al concepto de tecnología social como significación nueva y en construcción continúa siendo nuestro motor de marcha, de exploración y búsqueda, como así también el instrumento necesario para el desarrollo socio-económico, político y cultural desde lo local. En la actualidad, el equipo de investigación mantiene vínculos de trabajo con un grupo de investigadores de la Universidad de Quilmes-Buenos Aires, cuyo tema de investigación está centrado en tecnologías sociales. El vínculo permite el intercambio tanto teórico (marcos teóricos, marcos analíticos conceptuales, metodologías, epistemologías), como también empírico, y fortalece de manera singular el proceso de investigación para la construcción de nuevo conocimiento.

El proyecto PID 23121, Circuito Productivo Interactoral de casa partes de madera, finalizó en el año 2009. Dicho cierre se llevó a cabo a partir de un evento en el que participaron diversos actores de la comunidad, y cuyo objetivo fue presentarle a toda la comunidad presente las acciones realizadas en torno del proyecto. De esta manera, el evento contó con la presencia de todos los actores involucrados en las distintas etapas, tanto locales como nacionales. Se realizó en el marco de una demostración alternativa para posibles procesos habitacionales que se integran a acciones colectivas para el desarrollo local, con-

7- Tema de tesis doctoral: "Modelo tecnológico de gestión y producción interactoral para el desarrollo local a partir de la innovación tecnológica en Hábitat". Dicho doctorado se encuentra inscrito en el marco de los proyectos aquí presentados. 


\section{Artículos Arbitrados}

CUADERNO URBANO. Espacio, Cultura, Sociedad - VOL. 9 - № 9 (Octubre 2010) pp. 169-191. ISNN1666-6186

8- PROCODAS: Programa Consejo de la Demanda de Actores Sociales.

9- ANPCyT: Agencia Nacional de Promoción Científica y Tecnológica.

10- UBA: Universidad de Buenos Aires. siderando diversos aspectos como: uso y diversificación de los recursos locales, humanos y naturales, generación de empleo y emprendimientos productivos, generación de redes interactorales, generación de redes sociales solidarias, dinámica económica a partir del movimiento del mercado local, uso de tecnología social de manera co-construida a partir del saber diferenciado, entre otros aspectos. Participaron de este evento el municipio y todos sus actores, la escuela técnica, los productores forestales, los aserraderos, las familias beneficiarias, equipo de investigación de CEVE y vecinos de la localidad. También contó con la participación de autoridades del PROCODAS ${ }^{8}$; $\mathrm{ANPCyT}^{9}, \mathrm{UBA}^{10} \mathrm{y}$ directivos de Concordia y Ubajay.

En la actualidad el equipo de investigación procura aumentar la escala de intervención del proyecto a partir de la generación de una red interregional que articule a la localidad de Villa Paranacito con otras de la provincia de Entre Ríos; localidades con características similares en cuanto producción forestal y capacidades instaladas. Intentar escalar el Circuito Productivo es el desafío que asumimos en el presente, siempre acompañados por la búsqueda de modelos no excluyentes que permitan una mejora en la calidad de vida de toda la población.

\section{BIBLIOGRAFÍA}

ALFARO, Salvador (2009). "Gramsci y la sociología del conocimiento: un análisis de la concepción del mundo de las clases subalternas". En: http://www.uca.edu.sv/facultad/chn/ cll70/alfarol.html

CASAUBON (2006). En: "Diseño de matrices poliméricas biodegradables para la liberación controlada de feromonas en el control de insectos plaga: Megaplatypus mutatus (taladro de los forestales". Tesis Doctoral en el marco del proyecto PICT 670.

CORAGGIO, José Luis (2004) De la emergencia a la Estrategia. Más allá del alivio de la pobreza. ESPACIO, Buenos Aires.

CORAGGIO, José Luis (2004) La gente o el capital. Desarrollo local yeconomía del trabajo. ESPACIO, Buenos Aires.

DAGNINO, Renato (2004) Tecnología Social. Uma estrategia para o desenvolvimento. CIP, Rio de Janeiro.

PEYLOUBET, Paula (2008). "Tecnología Social. Tecnología para la inclusión social en 
Peyloubet, P. Massuh, H. O’Neill, T. Fenoglio, V. Valladares, G. DESARROLLO LOCAL A PARTIR DEL USO DE TECNOLOGÍA SOCIAL: UN ENFOQUE ALTERNATIVO

el marco del desarrollo local". Formulación de Proyecto PICT 2008 MINCYT-ANPCYTFONCYT.

PICT 670 ANPCYT (2007-2009) "Estudio teórico empírico de especies maderables no convencionales para su aplicación en la construcción de vivienda. Caso: álamo". Directora: Paula Peyloubet. Proyecto en curso.

PID 23121 ANPCYT (2007-2009) "Diseño e implementación de un circuito productivo interactoral de casa partes de madera que contribuya al fortalecimiento del perfil productivo del lugar, en el marco del desarrollo local". Caso: Villa Paranacito. Pcia. de Entre Ríos. Director: Héctor Massuh. Co-Directora: Paula Peyloubet. Proyecto finalizado.

THOMAS, Hernán (2009). "De las tecnologías apropiadas a las tecnologías sociales. Conceptos / Estrategias / Diseños / Acciones". Ponencia presentada al IV Seminario Iberoamericano de Ciencia y Tecnología organizado por el Centro Experimental de la Vivienda Económica del 23 al 25 de septiembre de 2009, en la ciudad de Córdoba, Argentina. VASILACHIS DE GIALDINO, Irene (2006) Estrategias de investigación cualitativa. Gedisa, Barcelona, España. 
|

$\overline{\overline{7}_{1}}$

$\mid \vec{\Gamma}$ 